\section{Letter: Safety Considerations for Neurosurgical Procedures During the COVID-19 Pandemic}

To the Editor:

The highest viral load for severe acute respiratory syndrome coronavirus 2 (SARS-CoV-2) is found in the lungs, nasopharynx, and oropharynx. Thus, working in close proximity to the face likely represents the greatest risk of SARS-CoV-2 exposure for neurosurgeons, many of whom have contracted and/or died from COVID-19. ${ }^{1}$ However, sources of exposure other than the respiratory tract should also be considered. SARS-CoV-2 viremia has been reported. ${ }^{2}$ SARS-CoV (which is closely related to SARS$\mathrm{CoV}-2$ ) can invade the brain. SARS-CoV-2 ribonucleic acid has been detected in the cerebrospinal fluid (CSF) of COVID-19 patients with altered mental status, intracranial hypertension, and/or brain imaging abnormalities, raising the possibility of SARS-CoV-2 meningitis or encephalitis. ${ }^{3}$ Thus, blood, brain, and CSF should be treated as potentially infectious until proven otherwise.

We conducted an internet-based search using the key words SARS-CoV-2, coronavirus, COVID-19, neurosurgery, and surgery using PubMed and Google. We also participated in a multispecialty task force to formulate evidence-based protocols for reducing the risk of SARS-CoV-2 exposure during neurosurgical operative procedures performed at our institution. Factors that may contribute to the risk of SARS-CoV-2 exposure during neurosurgical procedures were identified and used to construct a framework for risk stratification of SARS-CoV-2 exposure.

During the COVID-19 pandemic, only emergent or urgent cases should be performed in order to preserve resources for the influx of COVID-19 patients and reduce the SARS-CoV2 exposure risk associated with operating electively on patients harboring the infection. COVID-19 patients are considered a high risk for transmission of infection during surgical procedures. However, up to $87 \%$ of patients infected with SARS-CoV2 can be asymptomatic, infectious carriers. ${ }^{4}$ Thus, all patients undergoing surgical procedures should be tested preoperatively if possible. Unfortunately, the false negative rate of nasopharyngeal swab tests can be significant. Consequently, some institutions assume that all patients could be SARS-CoV-2 positive and universally adopt measures to prevent the transmission of infection.

Intubation and extubation pose significant risks of generating virus-laden aerosols. Breathing, talking, coughing, or sneezing can also produce airborne droplets containing SARS-CoV-2. Thus, awake neurosurgical procedures where the patient's airway is not controlled represent a high risk for SARS-CoV-2 exposure. To minimize the risks associated with airway aerosol generation, awake neurosurgical procedures should be avoided if possible. Intubation/extubation should be performed in rooms with negative pressure ventilation if available. If intubation or extubation must be performed in an operating room with positive pressure ventilation, a high efficiency particulate air filter can be placed near the patient's head to reduce droplet dispersal. Only anesthesia personnel should be in the room during intubation and extubation.

Transnasal, transoral, frontal sinus, or transmastoid approaches that traverse the sinorespiratory tract to reach the anterior skull base, sella, odontoid, clivus, or middle fossa are especially highrisk procedures. Procedures that involve exposure of the sinuses such as repair of frontal sinus fractures, subfrontal skull base approaches, or transmastoid approaches may expose the neurosurgeon to a high viral load. During the pandemic, transcranial approaches that do not traverse the sinorespiratory tract should be selected whenever possible.

Aerosolized droplets containing viable SARS-CoV-2 can remain suspended in the air for $3 \mathrm{~h}$ and can survive on surfaces for days. ${ }^{5}$ Thus, activities that aerosolize blood, bone, CSF, or brain (eg, the use of power drills, ultrasonic cavitating devices, forceful irrigation, or monopolar electrocautery) could disperse viral particles into the air and should be minimized. Unnecessary operating room entry or exit should be minimized, and the room should be thoroughly sanitized after each surgery.

As the duration of the neurosurgical procedure increases, the SARS-CoV-2 exposure risk also increases. Thus, the procedure duration should be kept to a minimum.

Throughout the surgical procedure, all personnel should be equipped with gloves, head and shoe coverings, impermeable gowns, face shields, N95 respirator masks, and/or powered airpurifying respirators (PAPRs), depending upon the level of exposure risk associated with the specific neurosurgical procedure. We recommend that PAPRs be used in combination with N95 masks for procedures with a high risk of SARS-CoV-2 exposure. PAPRs may cease to function after long neurosurgical procedures due to battery depletion. The use of an N95 respirator mask with the PAPR allows the PAPR battery and hose to be changed during the surgical procedure while providing a margin of safety.

Recognizing and mitigating SARS-CoV-2 exposure risks should improve the safety of neurosurgical procedures and preserve the health of the neurosurgical team.

\section{Disclosures}

The authors have no personal, financial, or institutional interest in any of the drugs, materials, or devices described in this article.

Rrita Daci, MD

Sabareesh K. Natarajan, MD, MS

Mark D. Johnson, MD, PhD

Department of Neurological Surgery

University of Massachusetts Medical School 
UMass Memorial Health Care Worcester, Massachusetts

\section{REFERENCES}

1. https://www.medscape.com/viewarticle/927976\#vp_3. Last Accessed April 12, 2020.

2. Chang L, Zhao L, Gong H, Wang L, Wang L. Severe acute respiratory syndrome coronavirus 2 RNA detected in blood donations. Emerg Infect Dis. 2020; 26(7):1-2.

3. Moriguchi T, Harii N, Goto J, et al. A first case of meningitis/encephalitis associated with SARS-coronavirus-2. Int J Infect Dis. 2020;94:55-58.
4. Sutton D, Fuchs K, D'Alton M, Goffman D. Universal screening for SARSCoV-2 in women admitted for delivery. $N$ Engl J Med. published online: 2020 (doi:10.1056/NEJMc2009316).

5. van Doremalen N, Bushmaker T, Morris DH, et al. Aerosol and surface stability of SARS-CoV-2 as compared with SARS-CoV-1. N Engl J Med. published online: 2020 (doi:10.1056/NEJMc2004973).

Copyright (C) 2020 by the Congress of Neurological Surgeons

10.1093/neuros/nyaa196 